\title{
Perceptual training to increase drivers' ability to spot motorcycles at T-junctions
}

David Crundall*, Aaron Howard and Angela Young

Division of Psychology

School of Social Sciences

Nottingham Trent University, UK

* Address for correspondence:

Prof. David Crundall

Division of Psychology

School of Social Sciences

Nottingham Trent University

Burton Street

Nottingham NG1 4BU

e-mail: david.crundall@ntu.ac.uk

Keywords: motorcycles, dual drivers, motorcycle experience, $T$-junctions, perceptual training, Pelmanism 


\begin{abstract}
Motorcyclists too often collide with other road users who pull out of side roads in front of them. These other road users typically report making all the necessary visual checks, despite failing to see the approaching motorcycle. These Look But Fail To See errors appear to be attenuated in road users who themselves have motorcycling experience, suggesting that motorcycle exposure may lower thresholds for spotting these vulnerable road users through natural perceptual learning. This raises the possibility that perceptual training could improve car drivers' abilities to spot motorcycles. Two experiments are reported. The first experiment demonstrated that a T-junction task, requiring participants to detect an approaching vehicle in briefly displayed images, was sensitive to participants' motorcycle experience, with dual drivers (who both ride motorcycles and drive cars) performing better than average car drivers. Following this, a second experiment split the car drivers into 2 groups. One group undertook a Pelmanism task requiring participants to match pairs of motorcycles, while the control group had to match pairs of fruit. When the two groups were retested on the T-junction task, the group who had undergone perceptual training for motorcycles via the Pelmanism task, were better able to identify approaching motorcycles, but not approaching cars. The results suggest that gamification of perceptual training for motorcycle detection provides a novel opportunity to improve driver safety.
\end{abstract}

\title{
INTRODUCTION
}

Motorcyclists are over-represented in collision and fatality statistics across the globe. In the UK, efforts to reduce motorcyclist fatalities have stagnated over the past 5 years, with 365 recorded fatalities occurring in 2015 , and over 5000 people seriously injured. When controlling for exposure the problem is evident: motorcyclists had an estimated 6,671 injuries (and 122 fatalities) per billion passenger miles, while car users had a mere 273 injuries (and 1.8 fatalities) per billion passenger miles. Even compared to other vulnerable road users such as pedestrians and cyclists, the fatality rate is staggeringly high (Department for Transport, 2016). This increased risk is reflected across Europe (EC, 2015), and many other countries including America (NHTSA, 2007), Australia (Johnston, Brooks \& Savage, 2008), China (Chang et al., 2016), and New Zealand (Walton, Buchanan \& Murray, 2013).

While a substantial number of collisions are single-vehicle crashes that may involve excessive speed or loss of control, the majority of incidents typically involve another vehicle failing to give way to a motorcycle at a junction (ACEM, 2009; Clarke et al., 2007). Such incidents may not be the fault of the motorcyclist, and can result in particularly severe injuries (Pai \& Saleh, 2008). While vehicle-to-vehicle communication, or car-based sensors, may eradicate this problem in the future, we face the possibility of a 15-25 year delay before we have wide-spread systems (Cavallo et al., 2015; IEEE, 2012). Thus research is required to mitigate against these collisions in the meantime. 
Crundall et al., (2008a, 2008b, 2008c; 2012) suggested that there are at least three links in the behavioural chain that could cause drivers to pull out in front of oncoming motorcycles: failures to look, failures to perceive, and failures to correctly appraise the risk posed by an approaching motorcycle. Failures to perceive are often termed 'Look But Fail To See' errors (Brown, 2002). These errors are hypothesised to occur when fixated information does not reach conscious awareness. Crundall et al. (2008b) discussed a range of factors that may underlie such errors, including the preference of the visual system for processing global, lowspatial frequency objects (e.g. cars and other wide vehicles) before interrogating high-spatial frequency items (e.g. motorcycles). A quick glance down the road might therefore miss an approaching motorcycle in the cluttered, high spatial frequency background of distant objects. Although this initial glance could be followed up with more deliberate visual search, the typical driver may well skip this step because of a low expectation for the presence of a motorcycle due to limited motorcycle exposure (e.g. Hills, 1980; Oliva \& Torralba, 2006). Following this argument, a driver may look directly at a motorcycle, but because they are expecting to see either a car or an empty road, they terminate the glance once they have processed only the low-spatial frequency information and are convinced that there are no approaching cars.

Interventions have tried to decrease collisions by targeting all three levels of failure. In regard to failures to look, Summala et al. (1996) concluded that speed-reducing countermeasures, such as speed humps, slowed Finnish drivers' approach speeds and gave them time to scan more appropriately for two-wheeled vehicles (in this particular case they were concerned with cyclists). Failures to perceive are typically targeted with studies designed to increase the sensory and cognitive conspicuity of riders. Sensory conspicuity interventions have focused on headlight saliency (Ranchet et al., 2016; Pinto et al., 2014) and rider clothing (Shaheed et al., 2015), while attempts to increase riders' cognitive conspicuity include targeting drivers with safety messages (Roge et al., 2015), and explicitly directing them to find motorcycles (Gershon et al., 2012). Failures of appraisal, such as the size-arrival effect, have also been targeted by headlight configurations designed to make it easier to extract optical expansion information from approaching motorcycles (e.g. Cavallo et al., 2015; Gould, et al., 2012 a, b).

\section{Exposure increases cognitive conspicuity}

The focus of the current study is to investigate a novel way of increasing the cognitive conspicuity of motorcyclists for car drivers. This stems from mounting evidence which suggests that exposure to motorcycles increases the ability to recognise them on the road, 
potentially reducing Look But Fail To See errors. Such studies suggest that when motorcyclists are behind the wheel of a car, they are less likely to crash into a motorcycle than average drivers (Magazzù et al., 2006; cf. de Craen et al., 2014), and that even nonriders who have close friends or relatives who ride motorcycles, and have had the opportunity to ride pillion, are better able to detect motorcycles (Brooks \& Guppy, 1990). This was further investigated by Crundall, Crundall, Clarke and Shahar (2012) who presented car drivers with video clips, taken from the driver's perspective, of a car approaching a T-junction from a minor road. Upon reaching the T-junction the participant had to press a button to signal when they felt it was safe to pull out. The image shown to participants spanned nearly 180 degrees of the filmed scene and was presented across three screens allowing participants to look to the left and right into the junction. Across 30 clips, drivers were faced with either clear roads (allowing an immediate pull-out response), or with a conflicting car or motorcycle (requiring a delayed pull-out response). Of all the participants who were tested, those drivers with experience of both driving cars and riding motorcycles ('dual drivers') gave the safest responses at junctions, especially when faced with an approaching motorcycle. It appears that this safety benefit was related to the dual drivers' eye movements. While they were no faster to fixate an approaching motorcycle than other car drivers, their initial gaze and mean gaze duration was longer on the motorcycles than was the case for other drivers. Their gazes on motorcycles were also longer than their corresponding gazes on cars. This is understandable: as motorcycles are smaller and less predictable than a car, one should expect them to evoke longer gazes indicative of the additional processing demand.

Experienced car drivers (without motorcycle experience) were, however, more likely to give longer first gazes to cars than motorcycles, perhaps indicating that in some instances they had fixated the approaching motorcycle but had not registered anything more than a clear road (at least in the first gaze). The authors concluded that the results demonstrated dual drivers to be more attuned to the image of an approaching motorcycle, and were therefore able to recognize the threat and allocate additional attentional resources.

A natural extension of the exposure argument is that drivers who live in areas with high motorcycle traffic levels should be better able to detect approaching motorcycles, regardless of their personal motorcycle riding experience. This hypothesis was tested by Lee, Sheppard and Crundall (2015) who compared Malaysian drivers and UK drivers on their ability to spot motorcycles. Powered two-wheelers make up the majority of registered vehicles in Malaysia, so one might assume that the average Malaysian driver is more exposed to motorcycles than an average UK driver. Using a methodology developed by Crundall et al., (2008c), they presented still images of Malaysian and UK junctions that contained a clear road, or either an approaching car or an approaching motorcycle at varying distances (essentially a simpler, 
static version of the more dynamic stimuli used by Crundall, et al., 2012). In the first experiment Lee et al. (2015) presented these pictures for only $250 \mathrm{~ms}$, after which participants were asked to report whether or not there was an approaching vehicle. They found evidence that, while all drivers were worse at spotting motorcycles than cars at a far distance, the detection accuracy gap between these vehicles was smaller for Malaysian drivers. Unfortunately, a second study found that this did not necessarily translate into road safety benefits as the Malaysian drivers were more likely to believe it was safe to pull out in front of the approaching vehicles in the images. This result may be an example of the double-edged nature of exposure: while it may lower thresholds for detecting certain stimuli, it can also desensitize one to the risks they pose.

\section{Perceptual learning and perceptual training}

It is not surprising that increased exposure to motorcycles improves one's ability to detect and then process those stimuli. Decades of research in the field of perceptual learning have demonstrated that experience of interacting with stimuli "results in long-term changes in the perception of these stimuli, pointing to experience-dependent plasticity in the visual system", (Sagi, 2011, p1563). Much work has been undertaken assessing perceptual learning of lowlevel features using, for instance, Vernier acuity (Poggio et al., 1992), sine wave gratings (Fiorentini \& Berardi, 1981), texture patterns (Karni \& Sagi, 1991), and checkerboards (McLaren, 1997). Equally, however, there are many studies which demonstrate that individually-acquired experience with certain stimuli can demonstrate natural perceptual learning. For instance, Diamond and Carey (1986) found perceptual learning in dog experts for specific breeds in which they specialized. Other domains in which perceptual learning has been demonstrated include interrogation of x-ray images (Myles-Worsley et al., 1988; Sowden, Davies \& Roling, 2000), bird discrimination (Tanaka \& Taylor, 1991), brand recognition (Qin, Koutstaal \& Engel, 2014), and even sexing new-born chicks (Biederman \& Shiffrar, 1987).

Many expert examples of perceptual learning reflect years of individual experience within a particular domain, and may explain the reported superiority of dual drivers over car drivers in spotting motorcyclists. While we cannot increase motorcycle safety by exposing individuals to years of motorcycle interaction, we could potentially induce perceptual learning via targeted training techniques to short-cut this experiential process (e.g. Gauthier \& Tarr, 1997; Husk, Bennett \& Sekular, 2007). 
Such training could target either the visual search of drivers, directing them to the correct locations, or the efficiency which drivers' process the approaching motorcycle once they have fixated it (Kellman, 2002). As Look But Fail To See errors suggest that the driver can look directly at an approaching motorcycle yet fail to identify it, we argue any training should focus not on where to look, but how to process the stimulus once one has looked at it.

Another feature of perceptual expertise that can guide the development of a training intervention is the robust finding that experts tend to be as fast at categorizing sub-ordinate classes of particular objects, as they are at categorizing the objects at a basic level (e.g. a dog-expert can classify a 'dachshund' as fast as a 'dog', while non-experts will be slower when categorizing at a sub-ordinate level). This effect holds with real-world and artificial stimuli (e.g. Tanaka \& Taylor, 1991; Gauthier \& Tarr, 1997). As basic-level processing is assumed to occur prior to sub-ordinate processing (Grill-Spector \& Kanwisher, 2005), one could predict that training in sub-ordinate classification should coincide with greater detection and identification of the base-level classifications (Harel, Ullman, Harari and Bentin, 2011; Hershler and Hochstein, 2009). There are even data to suggest that car experts, assessed on the basis of their ability to classify cars sub-ordinately, are better than non-experts at detecting the presence of cars in briefly presented natural scenes (Reeder, Stein \& Peelen, 2016). On this basis one might expect that successful training in sub-ordinate categories should not only improve subsequent sub-ordinate classification (Scott, Tanaka, Sheinberg \& Curran, 2006), but also improve detection of base-level exemplars in the real world.

The arguments presented above suggest that perceptual training for motorcycle detection should focus upon identification of the vehicle following fixation, and should involve discrimination between sub-ordinate classifications (i.e. different types of motorcycle). A third suggestion for the development of perceptual training for motorcycle detection, lies in the mode of presentation. There is evidence that a verbal, declarative approach to perceptual training may be less successful than a more visual and implicit approach (e.g. Jackson \& Farrow, 2005). This may be especially important when trainees are placed in high stress situations (Abernethy et al., 2012). On this basis, our training should involve visual stimuli which implicitly guide trainees to extract relevant information (a form of guided discovery learning; Magill,1998), rather than using explicit verbal instruction to direct trainees' learning.

Finally, one should consider the rationale on the learner's part for engaging with perceptual training. In some areas, such as elite sports (Abernethy et al., 2012) or developmental disorders such as amblyopia (Levi, Knill, \& Bavelier, 2015), there is a clear benefit for individuals to engage with training. However, in the domain of driver safety, individuals might be reluctant to voluntarily engage in a training intervention, particularly as most drivers 
already think they are better than the 'average' driver (e.g. Roy \& Liersch, 2014). One approach that is increasingly being used to improve voluntary engagement with training materials is gamification. This is described as the use of game elements in non-game contexts to achieve some goal, such as training (see Seaborn \& Fels, 2015, for a critical review). Under the appropriate conditions, gamification of tasks and situations can increase participant engagement with training materials, and it offers a potential route to gently train the perceptual skills of drivers without threatening their self-image.

Taken together, these arguments provide some clear guidelines for developing a potential perceptual training intervention for detecting motorcycles. First, it should concentrate on processing the motorcycle once fixated. Secondly, it should train at a sub-ordinate class level in order to maximize discrimination. Thirdly, the training should be visual, allowing participants to discover their own ways of discriminating between sub-ordinate categories, rather than via verbal prompts. Finally, the potential for individuals to voluntarily engage in this training would be improved if the training could be gamified.

One approach that fulfils all of these criteria is that of Pelmanism, a card game based on matching pairs of images (reportedly derived from the training materials provided by the Pelman Institute for the Scientific Development of Mind, Memory and Personality over a century ago; see www.ennever.com/histories/history386p.php). A player turns over successive pairs of cards from an array of face down cards, seeking to match the revealed images. If a pair of cards does not match, they are turned faced down again, and a new pair is selected. If played alone, one can score the game on the speed and number of turns it takes to clear the array of paired images. This game ostensibly tests spatial memory for incorrectly paired images, and has been used to demonstrate spatial memory advantages for pictures of evolutionally-relevant threat compared to more modern threats (Wilson, Darling \& Sykes, 2011). For the current paper, however, we are more interested in harnessing any perceptual learning that occurs with the discrimination that is required between the pairs of images.

\section{The current study}

Before we can assess the effects of perceptual training we need a test of motorcycle detection that is sensitive to the perceptual expertise of motorcyclists. We have selected the static image test used by Crundall et al., (2008c) and Lee et al., (2015). Both studies demonstrated that average car drivers are worse at detecting motorcycles than cars, while the latter study suggests that natural exposure to motorcycles increases one's ability to detect them. Although the preceding research would suggest that dual drivers (with 
motorcycling and car driving experience) would be superior at detecting motorcycles in this test, there has not been any study to demonstrate this.

The first experiment is therefore a partial replication of Crundall et al., (2008c) to identify whether this test is sensitive to the perceptual expertise of drivers with extensive motorcycle experience. We predict that dual drivers will be better at detecting approaching motorcycles in this test than car drivers with no motorcycling experience.

Once the sensitivity of the test to motorcycle experience is established, the second experiment will train half of our car drivers to discriminate between a variety of motorcycles using a motorcycle-themed Pelmanism test. The remaining car drivers will form a control group who will receive Pelmanism training in discriminating between different fruits. We predict that motorcycle Pelmanism training will improve motorcycle detection in a postintervention test using the approaching vehicle images of Crundall et al., (2008c) and Lee et al. (2015).

\section{Experiment 1}

The static image test of approaching vehicles used by Crundall et al. (2008c; experiment 1) was tested on two groups of drivers: average car drivers, and dual drivers with experience of both driving cars and riding motorcycles, in order to test its validity. It was necessary to establish whether this test could discriminate between levels of motorcycle exposure, before it could be applied it to the measurement of motorcycle-based perceptual training effects in experiment 2. We predicted that all participants would be worse at spotting motorcycles than cars, especially at far distances from the T-junction depicted in the images, though the repeated exposure of dual drivers to motorcycles should lower their thresholds for detecting motorcycles, presumably via natural perceptual learning, thus allowing them to detect more approaching motorcycles than car drivers.

\section{Method}

Participants. Thirty car drivers and 30 dual drivers were recruited for the study. The car drivers had a mean age of 33.8 years, with an average of 15 years since passing their test, and a mean of 7160 miles per year (17 males). The dual drivers had a mean age of 40.0 years (30 males), with an average of 20 years since passing their car driving test (8250 miles per year), and 19 years since passing their motorcycle test (3477 miles per year). Age, driving experience and annual car mileage were compared across the two groups yet none were found to differ significantly (all $p s>$.147). 
Design. A $2 \times 2 \times 3$ mixed design was employed. The between-groups variable was driver group (car drivers versus dual drivers). The first within-groups variable was the type of approaching vehicle displayed in the image (either a car or a motorcycle). The second within-groups variable was the distance that the vehicle appeared at from the viewer in each image (either at a near, intermediate or far distance). These conditions were reflected evenly across 60 presented images using 10 junctions. In addition to images of approaching vehicles, sixty empty roads (6 repetitions of each empty junction) were also presented. All stimuli were presented randomly within a single block. The primary dependent variable of interest was participants' accuracy at reporting whether an approaching vehicle was present or not, though response times were also recorded as a secondary measure.

Stimuli. Ten colour pictures were taken for this study of T-junctions in Nottinghamshire and Derbyshire (4032 x 3024, presented at $26 \mathrm{~cm} \times 20 \mathrm{~cm})$. A mix of urban and rural junctions were included, photographed from the point-of-view of a driver who has pulled up to the give-way line of a side road, and is looking to the right to assess whether it is safe to pull out. Following the approach used by Crundall et al., (2008c), cars and motorcycles were edited into these photographs. Each junction was edited to contain both a motorcycle and a car at each of three distances (near, intermediate and far, see figure 1) producing 7 versions of each junction ( 1 empty junction, 3 with motorcycles at each distance and 3 with cars at each distance). While different motorcycles and cars were used across the 10 junction images, within the set of 3 motorcycle and 3 car images for each junction, the same vehicles were used, differing only on scale and location within the scene. For details on the percentage of the image that each vehicle covered please see Table 1. Approaching vehicles were scaled to the same size at each level of vehicle and distance (i.e. all intermediate cars approximated $0.29 \%$ coverage of the overall image). 
Table 1. The size of the various approaching vehicles as a proportion of the overall image

\begin{tabular}{|c|c|c|c|c|c|}
\hline & & & & area & \\
\hline & & $x$ & $y$ & $\left(\right.$ pixels $\left.{ }^{2}\right)$ & $\%$ of image \\
\hline \multirow[t]{2}{*}{ Empty Road } & & 4032 & 3024 & 12192768 & \\
\hline & Near & 420 & 340 & 142800 & 1.17 \\
\hline \multirow[t]{3}{*}{ Car } & Int. & 210 & 170 & 35700 & 0.29 \\
\hline & Far & 105 & 85 & 8925 & 0.07 \\
\hline & Near & 180 & 348 & 62640 & 0.51 \\
\hline \multirow[t]{2}{*}{ Motorcycle } & Int. & 90 & 174 & 15660 & 0.13 \\
\hline & Far & 45 & 87 & 3915 & 0.03 \\
\hline
\end{tabular}
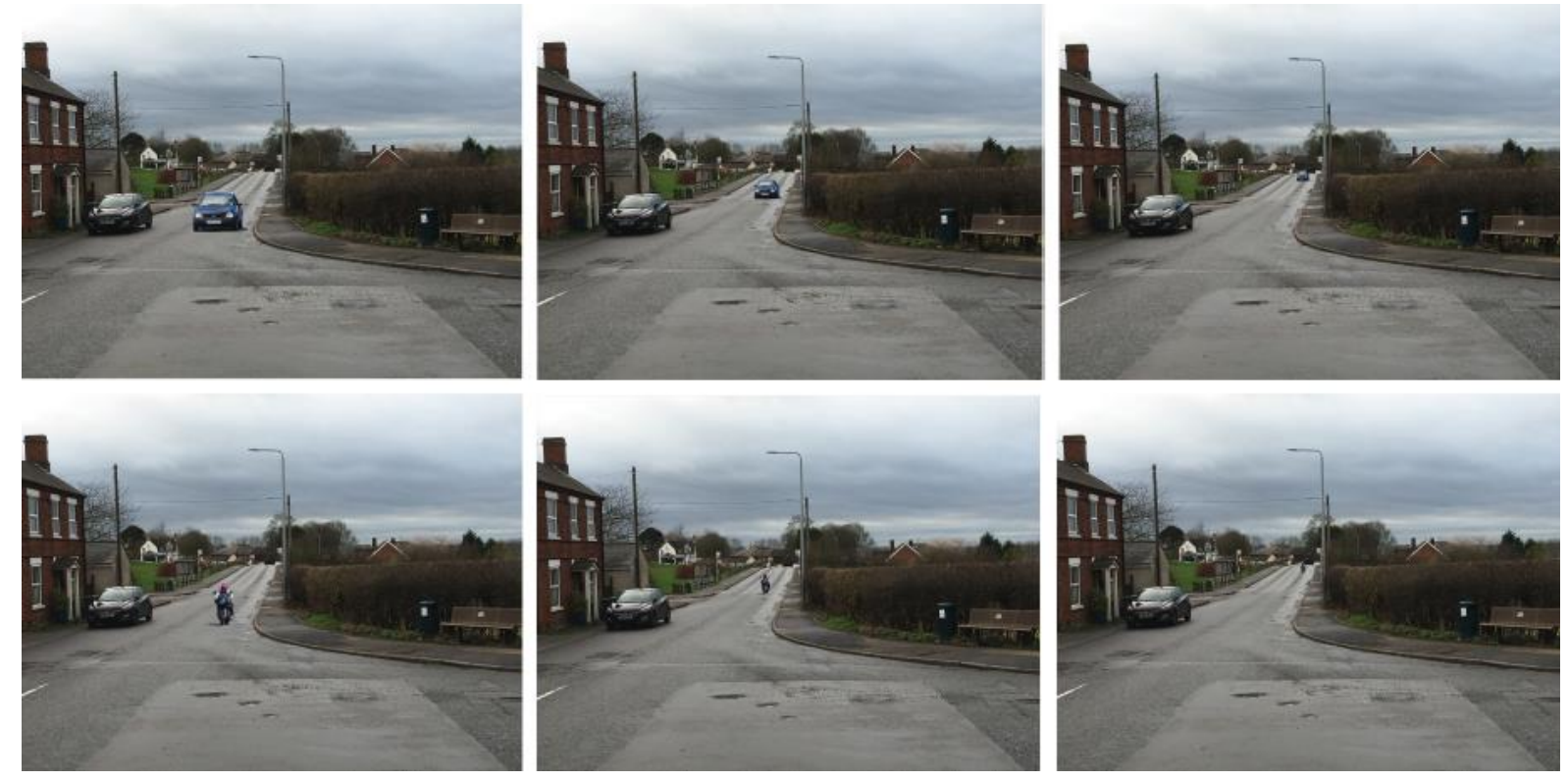

Figure 1: An example of a T-junction edited to include a motorcycle and car at far, mid and near distances.

Procedure. Testing was undertaken on a laptop at venues where motorcyclists were known to gather (e.g. particular cafés) or in private residences. Car drivers were recruited and tested in similar venues. Actual testing was conducted in a quiet area away from other people. All participants were seated at a table approximately $70 \mathrm{~cm}$ away from a laptop with a 
16:9 aspect ratio, with the images subtending 20.65 degrees along the horizontal axis, and 15.86 degrees in the vertical axis.

Participants were informed that they would see 120 road images taken from the perspective of a UK car driver looking right at a T-junction while waiting to pull out. They were told that some of the road images would be empty, while others would contain an approaching car or motorcycle at varying distances. Following a $500 \mathrm{~ms}$ central fixation cross, each picture appeared for $250 \mathrm{~ms}$. Following presentation, participants were told that they had two seconds to press one of two buttons on the keyboard ('m' or ' $z$ ') depending on whether they saw an approaching vehicle. It was made clear to participants that they were merely responding to the presence of an approaching vehicle rather than having to decide whether it was safe to pull out. A practice block of 10 trials was provided prior to the study, with images drawn randomly from the available stimuli. Trial-by-trial feedback was provided during the practice block regarding accuracy and reaction times, but was not available during the main study.

\section{Results}

All sixty participants provided data for the $2 \times 2 \times 3$ mixed design Analysis of Variance (ANOVA) comparing driver group (car vs. dual drivers), across approaching vehicle (car vs. motorcycle) at three distances (near, intermediate and far). Since the key question was whether this test could discriminate between those with different levels of motorcycle exposure, the main variable of interest was participant group. This produced a significant main effect, with dual drivers more accurately identifying the presence of an approaching vehicle than car drivers (89.4\% vs. 84.5\%; $\left.\mathrm{F}(1,58)=4.9, \mathrm{MSe}=146.9, \mathrm{p}<.05, \eta_{\mathrm{p}}{ }^{2}=.08\right)$. Participant group did not interact with any of the other factors.

In regard to the other independent variables, cars were identified more accurately than motorcycles (90\% vs 84\% accuracy; $F(1,58)=29.0$, MSe $\left.=88.3, p<.001, \eta_{p}^{2}=.33\right)$, and a main effect of distance was noted (with $90.3 \%, 91.1 \%$ and $79.5 \%$ reflecting near, mid and far accuracy, respectively; $\left.\mathrm{F}(2,116)=28.7, \mathrm{MSe}=174.6, \mathrm{p}<.001, \mathrm{n}_{\mathrm{p}}^{2}=.33\right)$. Repeated planned contrasts isolated this main effect to the comparison between the far distance and the mid distance $\left(F(1,58)=41.2, \mathrm{MSe}=391.1, \mathrm{p}<.001, \mathrm{\eta}_{\mathrm{p}}{ }^{2}=.42\right)$. Both of these withingroup main effects were subsumed by an interaction between vehicle and distance $(F(2,116)$ $\left.=12.2, \mathrm{MSe}=83.3, \mathrm{p}<.001, \eta_{\mathrm{p}}{ }^{2}=.17\right)$. Repeated contrasts identified the interaction to lie 
between the far and intermediate distances $\left(\mathrm{F}(1,58)=15.5, \mathrm{MSe}=162.6, \mathrm{p}<.001, \mathrm{n}_{\mathrm{p}}^{2}=\right.$ .21). As can be seen in Figure 2, while accuracy for spotting all vehicles was degraded at the far distance, motorcycle accuracy was degraded more than cars.

One problem with interpreting the main effect of driver group is that a simple analysis of hit rates for approaching vehicles could mask a criterion difference between the two groups (i.e. dual drivers might obtain higher accuracy for approaching vehicles because they have a greater tendency to respond positively regardless of the information available). To overcome this issue, we turned to Signal Detection Theory (SDT), and calculated d' (a measure of sensitivity to the signal; zHits - zFalse Alarms) and $c$ (the criterion, or propensity to say yes regardless of the information; (zHits $+z$ False Alarms)/2) for each participant (Stanislaw \& Todorov, 1999, and following Macmillan \& Kaplan's, 1985, suggestion for correcting for floor and ceiling effects). These measures combined the hit rate for each participant across all vehicles and distances and compared them to the number of false alarms, where participants reported an approaching vehicle at an empty junction.

An independent t-test compared these SDT measures across the two driver groups. Dual drivers were found to have greater sensitivity to approaching vehicles than car drivers $\left(t_{d^{\prime}}(58)\right.$ $=2.2, p<.05$, Cohen's $d=.61$; with d' of 2.7 and 2.2 , respectively), though there was no difference between the groups in terms of criterion $\left(\mathrm{t}_{c}(58)=1.0, p=.34\right.$; with criterion values of -0.04 and 0.03 , respectively). 


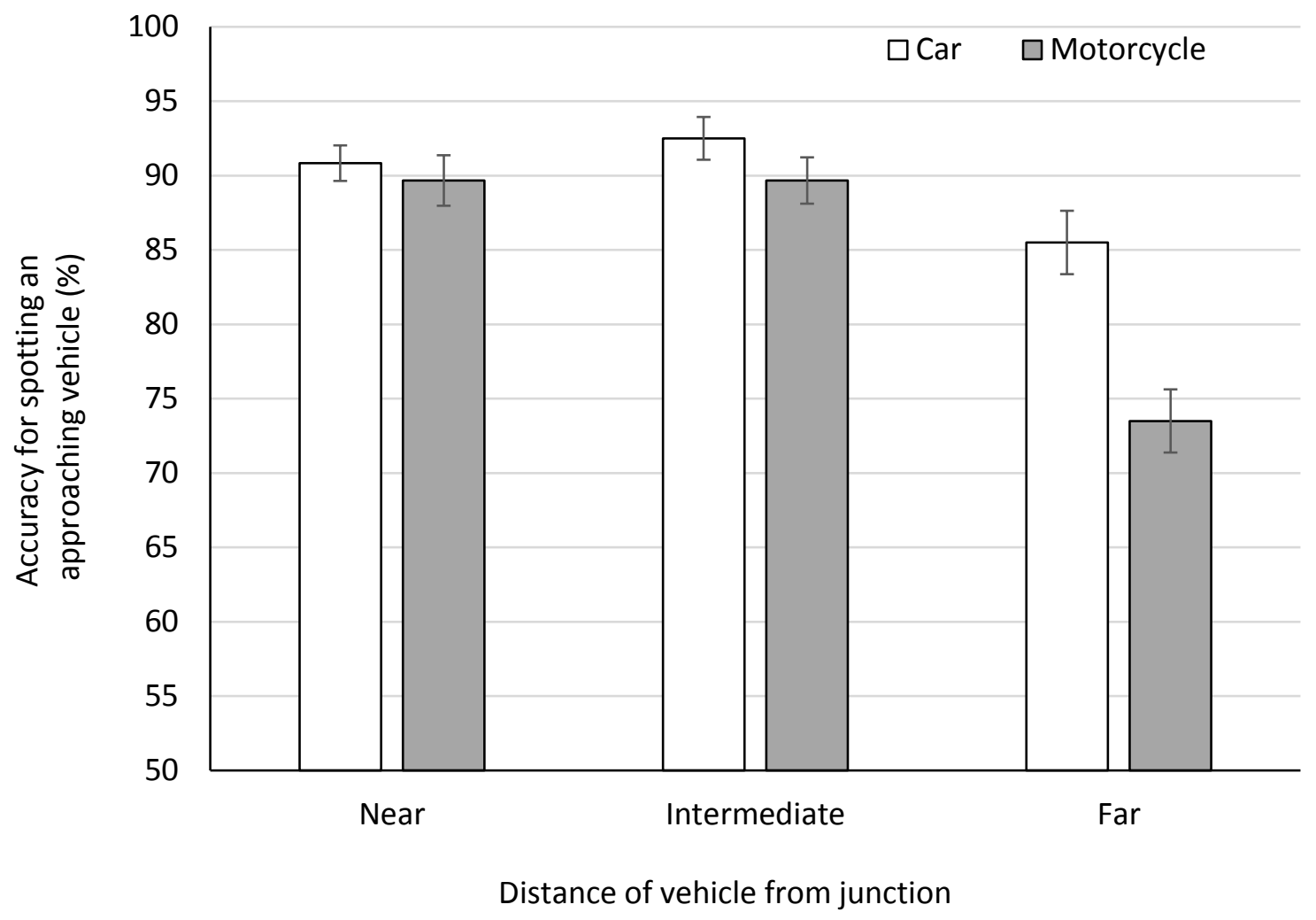

Figure 2. Percentage accuracy for correctly identifying the presence of an approaching vehicle across car drivers and dual drivers.

A $2 \times 2 \times 3$ ANOVA was also conducted on the response times of participants to correctly identify the presence of an approaching vehicle. Only distance produced a significant effect (with $577 \mathrm{~ms}, 591 \mathrm{~ms}$ and $656 \mathrm{~ms}$ reflecting response times to near, mid and far vehicles; $\left.F(2,116)=44.0, M S e=4827.4, p<.001 ; \eta_{p}^{2}=.43\right)$. Contrasts confirmed that far vehicles were responded to more slowly than intermediate vehicles (656 ms vs. $591 \mathrm{~ms} ; \mathrm{F}(1,58)=$ $\left.51.5, \mathrm{MSe}=9815.9, \mathrm{p}<.001, \mathrm{\eta}_{\mathrm{p}}^{2}=.47\right)$.

\section{Discussion}

The data have replicated the pattern found by Crundall et al. (2008c) and Lee et al. (2015), with motorcycles being especially difficult to spot when they appear far from the junction. More importantly, the between-group effect has demonstrated a superiority in the dual drivers. While it was predicted that this superiority would appear primarily for the approaching motorcycles, they demonstrated greater accuracy in detecting all approaching vehicles. Several studies have demonstrated that motorcyclists respond faster than car drivers to a variety of on-road hazards (Horswill \& Helman, 2003; Rosenbloom et al., 2011; Underwood \& Chapman, 1998), and, given their vulnerability, it is not surprising to find that they are sensitive to on-coming cars as well as approaching motorcycles. We have, 
however, ruled out the possibility that this may have occurred due to a criterion bias in our dual driver group.

There were, however, other ostensible differences between the two groups that may have contributed to the superior performance of dual drivers. While apparent gaps in age, experience and mileage across the two groups were not found to differ significantly, the imbalance of males and females poses a greater potential issue (the dual driver group was all male, while $43 \%$ of car driver group was female). Could this have confounded the results? To assess this we repeated the analysis comparing only the male car drivers $(N=17)$ to the dual driver group $(\mathrm{N}=30$ ), in terms of their accuracy at reporting an oncoming vehicle. The pattern of significant results was identical to that found with the whole sample, reducing the possibility that the sex imbalance confounded the results.

In conclusion, even though the beneficial effect of being a dual driver appears to stretch across both classes of approaching vehicle, this provides us with a baseline test against which we can compare the performance of ordinary car drivers following perceptual training in the discrimination of motorcycles.

\section{Experiment 2}

Having demonstrated the sensitivity of the T-junction test to motorcycling experience, the question remains whether training can short-cut the motorcycling experience that may improve one's ability to spot approaching vehicles. It is possible that the superiority of dual drivers for spotting approaching vehicles is due, in part, to their vulnerability on the roads (when riding a motorcycle) which may encourage greater sensitivity to all approaching vehicles, however the previous literature suggests that exposure is likely to have contributed to the better detection of motorcycles in this group.

The proposed intervention is based on the pair-matching game of Pelmanism, using images of front-facing motorcycles that require a level of sub-ordinate discrimination. As such, this intervention should only target the mechanism that supports riders' superiority at spotting other motorcycles, rather than cars.

To this end, the car drivers who were recruited for experiment 1 were randomly divided into a training group and a control group immediately following their contribution to Experiment 1. The training group were required to complete a number of Pelmanism arrays, pairing motorcycles. The control group undertook a similar task, though they were required to match pairs of fruit. It was predicted that the training group, exposed to the motorcycle Pelmanism 
task, would be better at spotting motorcycles in a subsequent T-junction task, whereas the fruit Pelmanism task would produce no benefit.

\section{Method}

Participants. For experiment 2, the car drivers from experiment 1 were randomly allocated to either a training group or a control group. The trained group consisted of 15 car drivers (mean age $=35.9$ years, 9 male). The car drivers allocated to the control group had a mean age of 32.7 years ( 8 male).

Design. A $2 \times 2 \times 2 \times 3$ mixed design was employed. The between-groups variable was driver group (trained drivers vs. control drivers), while the within-groups variables were approaching vehicle (car vs. motorcycle), distance of the approaching vehicle (near, intermediate, far), and time of testing (before or after the training/control intervention). In all other aspects, the design of the study was identical to experiment 1.

Stimuli. The T-junction stimuli remained the same as that in the first experiment, though new stimuli were created for the Pelmanism-based training and control interventions. The Pelmanism games were designed using a template program available from http://primary.naace.co.uk/activities/ . Players are presented with 24 virtual face-down cards on a laptop screen. Using the mouse cursor, a player must click on a card to reveal what is on the underside. Having viewed this image, the player then selects a second card to turn over, in the hope of finding an identical image. If the images do not match, both cards are turned back over, and this counts as one turn against their total score. The player can then select another two cards and so on. The lowest number of turns that a player would need to clear the array would be 12 (though such a low score would be an unlikely chance event, and would not involve any discrimination of images or need to memorise locations).

For the training intervention, twelve different images of motorcycles, photographed from the front, were sourced from the internet. The control intervention used the same game format, but used pictures of fruit rather than motorcycles. Each virtual game card measured $3 \times 3$ degrees of visual angle. Screen shots from the game are shown in Figure 3. 

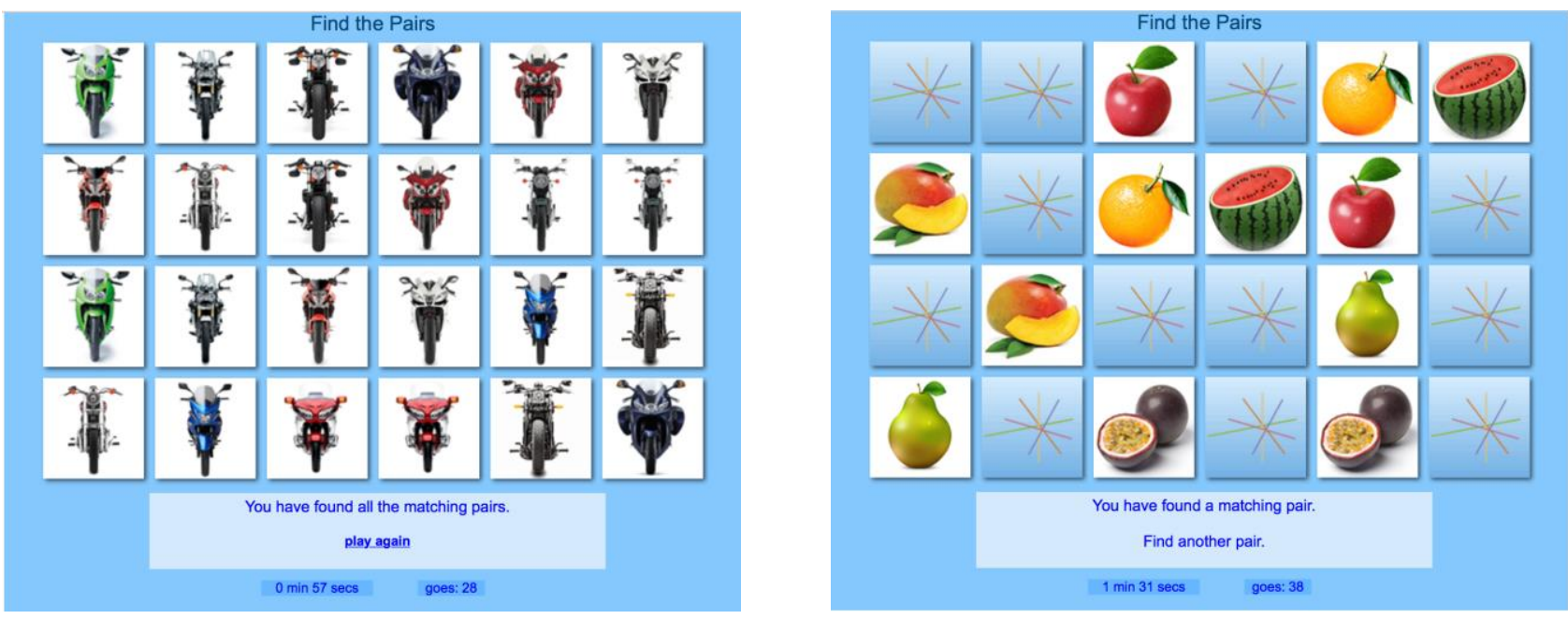

Figure 3: Two screen shots from the intervention. The left panel contains a completed game taken from the training intervention with 12 pairs of front-facing motorcycles. The right panel is from the control intervention, and reflects a partly-completed game with 6 pairs of fruit already identified.

Procedure. Immediately following the first experiment, car drivers were randomly allocated into the training intervention or control intervention group (without their knowledge) and received instructions for the Pelmanism game. They undertook 5 games of Pelmanism, consistently searching for 12 pairs or motorcycles (training group) or 12 pairs of fruit (control group). They were instructed to undertake the games as quickly as possible while trying to minimise the number of turns they took. Completing 5 games took approximately 6 minutes. Once the car drivers had successfully undertaken 5 games they completed the T-junction task once again.

\section{Results}

The data from one participant in the trained group was removed due to a software crash.

Data from the remaining 29 participants were entered into a $2 \times 2 \times 2 \times 3$ ANOVA comparing trained and control participants' $T$-junction performance both before and after the intervention, across vehicle type and distance of the vehicle from the junction.

As expected from experiment 1 , the main effects of vehicle and distance, and the interaction between the two, remained significant $\left(F_{\text {vehicle }}(1,27)=7.2, \mathrm{MSe}=182.3, \mathrm{p}<.05, \mathrm{n}_{\mathrm{p}}{ }^{2}=.21\right.$; 
$F_{\text {distance }}(2,54)=17.0, \mathrm{MSe}=318.2, \mathrm{p}<.001, \eta_{\mathrm{p}}^{2}=.39 ; \mathrm{F}_{\text {vehicle }} \mathrm{x}$ distance $(1,27)=6.7, \mathrm{MSe}=$ 115.0, $\left.\mathrm{p}<.005,, \mathrm{n}_{\mathrm{p}}^{2}=.20\right)$. Repeated contrasts again identified the interaction to lie between the far and intermediate distances $\left(F(1,27)=13.8, \mathrm{MSe}=196.0, \mathrm{p}<.001, \mathrm{n}_{\mathrm{p}}{ }^{2}=\right.$ .34).As can be seen in Figure 4, accuracy for spotting motorcycles once again suffers the most at far distances, as expected.

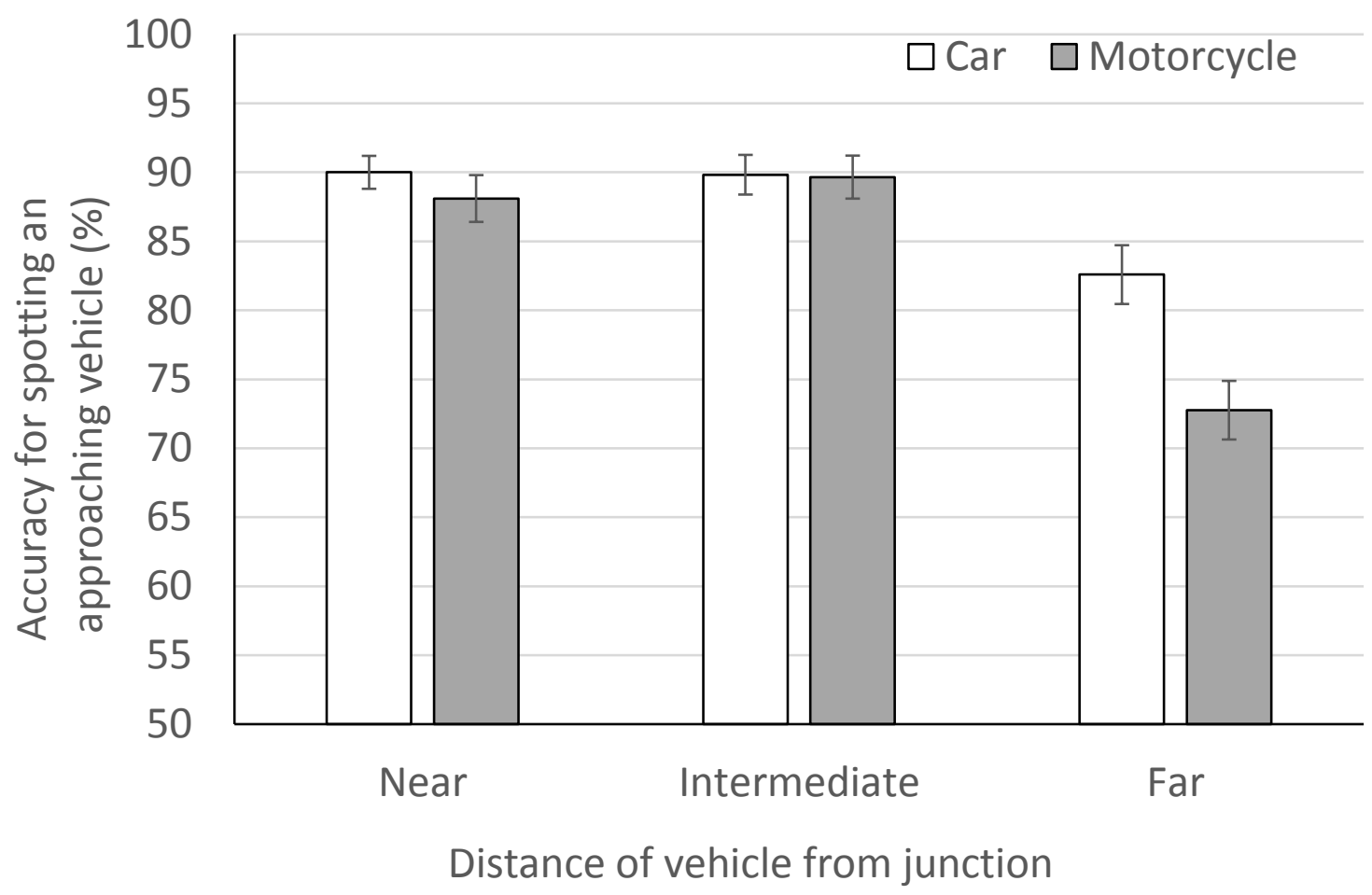

Figure 4. Percentage accuracy for correctly identifying the presence of an approaching vehicle across trained and control car drivers.

There was also a main effect of the time of testing $\left(F(1,27)=5.9, M S e=125.7, p<.05, \eta_{p}^{2}=\right.$ .18) with all participants (training group and control group) performing better following the intervention ( $84 \%$ vs. $87 \%)^{1}$. Finally a three-way interaction (Figure 5 ) was noted between time of testing, participant group (training or control) and vehicle type $(F(1,27)=4.5, \mathrm{MSe}=$ $\left.98.5, \mathrm{p}<.05, \mathrm{\eta}_{\mathrm{p}}^{2}=.14\right)$. T-test comparisons comparing before and after scores for each vehicle for each group revealed that the intervention improved accuracy for spotting motorcycles in the trained group (rising from $82.4 \%$ before the intervention to $88.3 \%$

\footnotetext{
${ }^{1}$ Sharp-eyed readers comparing Figure 4 to Figure 2 may note that performance levels do not appear to have improved across the two studies as indicated by this main effect. This is because Figure 2 includes data from the dual drivers in experiment 1.
} 
following the intervention; $\mathrm{t}(14)=2.8, \mathrm{p}<.05)$, while the control drivers demonstrated improvement in spotting cars (rising from $85.6 \%$ before the filler intervention to $90.0 \%$; $t(14)$ $=2.8, \mathrm{p}<.05)$.

As with experiment 1, measures of d' and c were calculated to assess whether the improvement in participants' scores from the first testing session to the second testing session (before intervention vs. after intervention) was due to a change in sensitivity to the signal of an approaching vehicle, or a shift in the participants' response criterion. These SDT measures were calculated for each participant for both the first and second testing sessions (collapsing across the vehicle and distance factors) and compared in a 2 × 2 ANOVA (before/after intervention $x$ trained/untrained group). Analysis of $d^{\prime}$ revealed a main effect of time of testing ( $d_{\text {'before }}=2.2 \mathrm{vs}$. $\mathrm{d}_{\text {after }}=2.5 ; \mathrm{F}(1,27)=8.1, \mathrm{MSe}=0.177, \mathrm{p}<.01, \mathrm{n}_{\mathrm{p}}{ }^{2}=.23$ ), but no effect of training group, and no interaction between the two factors. A similar analysis of the criterion failed to reveal any main effects or an interaction. This demonstrates that the effects noted in figure 5 are due to an increase in sensitivity for the signal of an approaching vehicle rather than a shift in participants' criterion, with the control group becoming more sensitive to the presence of cars, while the trained group become more sensitive to the presence of motorcycles. 


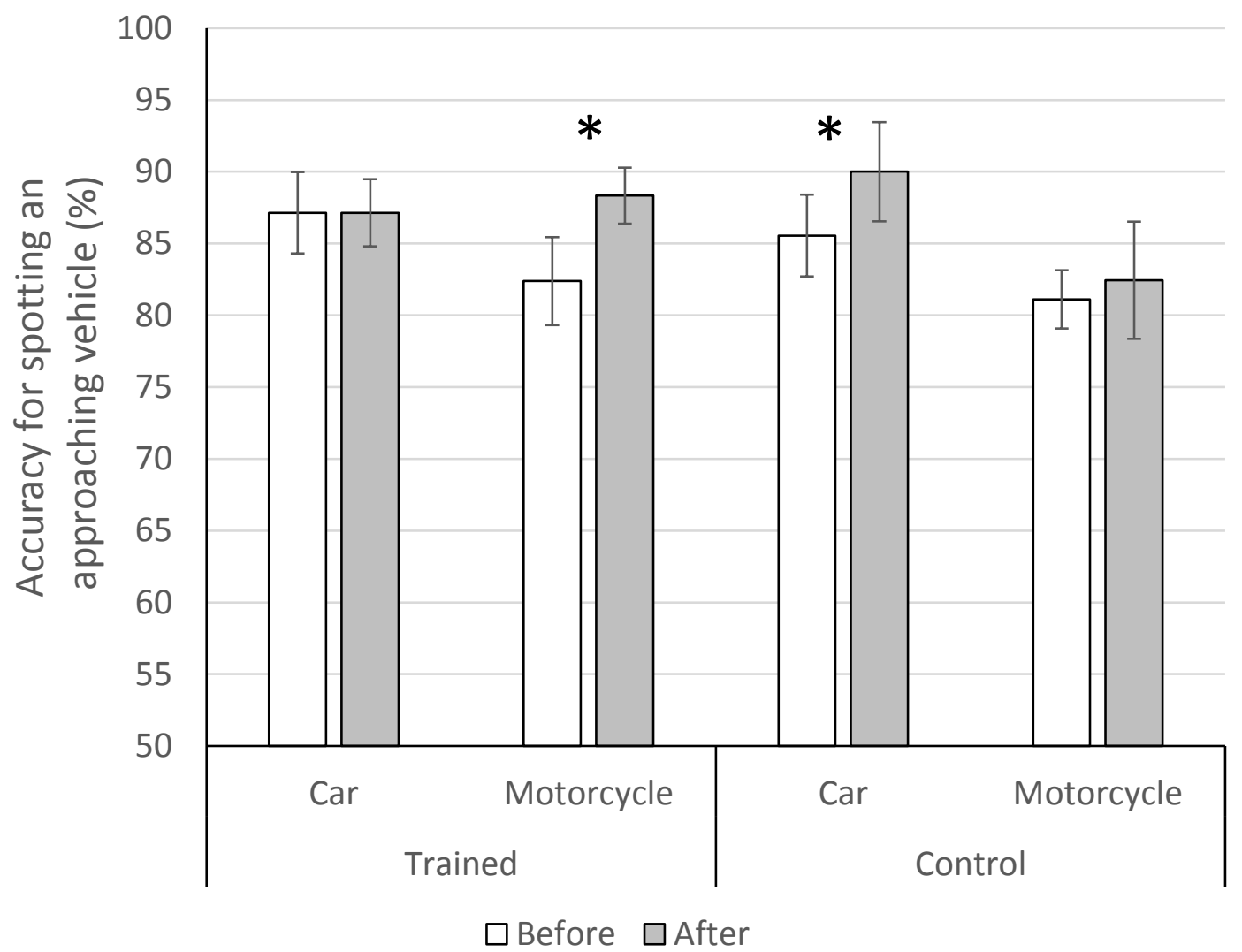

Figure 5. Percentage accuracy for correctly identifying the presence of an approaching vehicle before and after either the training intervention or the control intervention, with standard error bars added ( $\left.{ }^{*} p<.05\right)$.

A $2 \times 2 \times 2 \times 3$ ANOVA conducted on the response times revealed slower responses to distant vehicles $\left(F(2,54)=18.5, \mathrm{MSe}=9609, \mathrm{p}<.001, \eta_{\mathrm{p}}{ }^{2}=.41\right)$ and that all participants were faster following the intervention $\left(F(1,27)=15.7, M S e=30410, p<.001, \eta_{p}^{2}=.37 ; 610\right.$ ms vs. $535 \mathrm{~ms})$.

\section{Discussion}

The first experiment demonstrated that the T-junction task is sensitive to the experience of motorcycle riders (dual drivers) which is reflected in their ability to detect approaching vehicles in briefly presented images of naturalistic road scenes. This fits with previous studies that have demonstrated superior hazard perception skills for motorcyclists (Horswill \& Helman, 2003; Rosenbloom et al., 2011; Underwood \& Chapman, 1998), and with studies that have demonstrated perceptual expertise to improve detection of objects in natural scenes (e.g. Reeder, Stein \& Peelen, 2016). While it was predicted that dual drivers would excel at detecting motorcycles compared to average car drivers, it was found that they were actually superior at detecting all approaching vehicles. This effect was not simply due to a 
difference in response criterion, but appeared to be due to participants' sensitivity to the oncoming vehicles. The increased sensitivity to both cars and motorcycles is understandable from the perspective of a vulnerable road user, though it is possible that different underlying mechanisms lead to these benefits. While exposure (and correlated aspects of motorcycling experience such as knowledge and empathy; Crundall et al., 2008a) may be responsible for the perceptual learning associated with motorcycles, the cognitive conspicuity of the cars may related to the higher level of threat these vehicles pose when on the road.

The second experiment attempted to improve the ability of the car drivers to detect the approaching motorcycles with a Pelmanism game requiring them to match pairs of motorcycles. The control group matched pairs of fruit. In the post-intervention T-junction task, the control group improved in their ability to detect oncoming cars, but did not show improvement in the detection of motorcycles. This improvement is likely due to having practiced the T-junction task prior to the intervention, with the pattern of results explained by Ahissar and Hochstein (1997), who suggest that practice on simple visual tasks (i.e. spotting approaching cars) will lead to subsequent improvement, though more difficult tasks (i.e. spotting motorcycles) will require more a deeper or more active level of practice or training before learning benefits are noticed.

The group who received the motorcycle Pelmanism game significantly improved in their ability to spot motorcycles in the post-intervention T-junction test. This was predicted on the basis of perceptual learning for motorcycles at the sub-ordinate category that was provided in the intervention. While drivers were not required to classify the motorcycles with verbal labels, they were implicitly required to discriminate between them based on visual features which could have included width, colour, headlight configuration, handlebar shape, sidemirror design and windshield shape.

Interestingly, this benefit for motorcycle perception appears to have been gained at the expense of the, presumably practice-based, improvement for car detection noted in the control group. This suggests that perceptual training has overridden the practice benefit for detecting cars. One can view this in the same way that a disruption task might be used to prevent participants from rehearsing short-term memory items: by asking participants to undertake the motorcycle task, we are preventing them from consolidating task-learning (which would default to cars as the easiest and most expected stimulus in the scenes; Ahissar \& Hochstein, 1997). This would require cars and motorcycles to be represented differently within the visual system, otherwise training on motorcycles should also improve car detection (or at least not impede the practice-based improvement that would normally 
occur). The most obvious method for perceptually separating the processing of cars and motorcycles is via spatial frequency, which has already been put forward as a possible explanation for Look But Fail To See errors. An interesting parallel can be drawn with the study of Lee et al. (2015). They found that Malaysian drivers (who have higher exposure of motorcycles as compared to UK drivers) did not excel in perceiving motorcycles per se compared to UK drivers, but rather the gap between their ability to spot motorcycles and cars was significantly reduced. Similarly to the participants in the current study, it appears that the Malaysian drivers also demonstrated a trade-off in car detection for motorcycle detection.

Regardless of the reason for the lack of improvement in car detection following the motorcycle-based intervention, one might argue that this poses a problem for future attempts to improve motorcycle detection: Might we be recommending a task that makes drivers comparatively worse at detecting one type of road user in favour of another? Two points should be considered. First, car detection is typically much closer to ceiling than motorcycle detection. Even if motorcycle detection training led to decreased car detection, the value of increasing motorcycle safety may be greater than the costs associated with a slight decrease in car detection accuracy. Second, our drivers only became better at detecting cars in this study because of practicing the $\mathrm{T}$-junction task. If the intervention merely removes the benefit that would otherwise have accrued via practice on the T-junction test, then the training game should not reduce drivers' ability to detect cars beyond the level of ability which they had prior to undertaking the study.

Further research is required to first replicate this ostensible trade-off in perceptual learning, and, if the effect persists, to explore the conditions under which it occurs. It is possible that a different training schedule could retain any practice-based effects of the T-junction test, while maintaining or even improving the perceptual learning effect for motorcycles. For instance, creating a time gap between the initial $\mathrm{T}$-junction test and the subsequent intervention, may allow any practice effects to be consolidated before perceptual training begins.

Other avenues for future research include manipulating the views of the motorcycles used in both the training images and the post-intervention test (e.g. front view, side view, three quarters view) to assess how the angle of orientation of the paired images maps to the angle of the motorcycles detected in the natural scenes. For maximum impact, perceptual training should improve detection of objects from all angles. In the current T-junction test, the approaching motorcycles were not quite head-on, suggesting at least a modest transferal of learning from the paired images (which were all perfectly head-on, without a rider) to the 
natural scenes. It is possible that the orientation of the training stimuli remains relevant, however, and head-on paired images may offer no perceptual-training benefits for motorcycles that appear side-on, for example, in the real world. Other aspects of the motorcycles presented in the Pelmanism game (colour, make, headlight configuration, etc.) may also be important to the transfer of training. In the current study, there was no overlap between the types of motorcycle used in the training intervention and those images used in the post-intervention test, again suggesting that transference beyond the particular subordinate categories that one is trained in.

Finally, any training effects need to be measured using different post-intervention tests, potentially using more dynamic hazard perception clips involving motorcycles or using scripted scenarios in a driving simulator. Where possible the duration of the effect over a longer period should also be recorded. We would hope that a suitable training task could last sufficiently long enough to have an effect on participants' real world driving (Chapman, Underwood and Roberts, 2002).

In conclusion, we have provided the first evidence that brief Pelmanism-based perceptual training, using sub-ordinate classes of motorcycle, is sufficient to improve the average car driver's ability to spot motorcycles in natural scenes, using a post-intervention test that has been shown to be sensitive to motorcycle experience. This opens the way to online training games than can potentially encourage drivers to partake in perceptual training, targeted at the most vulnerable of road users. With the addition of other gaming elements (badges, scores, online norms, etc.), and the possibility of additional game mechanics designed to improve a variety of on-road safety related behaviours, we have the opportunity to provide continuous and appealing development opportunities for drivers.

\section{References}

Abernethy, B., Schorer, J., Jackson, R. C., \& Hagemann, N. (2012). Perceptual training methods compared: the relative efficacy of different approaches to enhancing sport-specific anticipation. Journal of Experimental Psychology: Applied, 18, 2, 143-153.

Ahissar, M., \& Hochstein, S. (1996). Learning pop-out detection: Specificities to stimulus characteristics. Vision Research, 36, 21, 3487-3500.

Association des Constructeurs Européens de Motocycle (ACEM) (2009). In-depth investigations of accidents involving powered two wheelers (MAIDS). Retrieved from http://www.maidsstudy.eu, on $17 / 10 / 16$. 
Biederman, I. \& Shiffrar, M. M. (1987). Sexing day- old chicks: a case study and expert systems analysis of a difficult perceptual-learning task. Journal of Experimental Psychology: Learning, Memory and Cognition, 13, 640-45.

Brooks, P. and Guppy, A. (1990) Driver Awareness and Motorcycle Accidents. Proceedings of the International Motorcycle Safety Conference, 2, 10, 27-56.

Brown, I.D. (2002). A review of the 'looked but failed to see' accidents causation factor. In: Behavioural Research in Road Safety XI, Department of Transport, Local Government and the Regions, London.

Cavallo, V., Ranchet, M., Pinto, M., Espié, F., Vienne, F., \& Dang, N. T. (2015). Improving car drivers' perception of motorcycle motion through innovative headlight configurations. Accident Analysis and Prevention, 81, 187-193.

Chapman, P. R., \& Underwood, G. (1998). Visual search of driving situations: Danger and experience. Perception, 27, 8, 951-964

Chapman P. R., Underwood G., \& Roberts K. (2002). Visual search patterns in trained and untrained novice drivers. Transportation Research Part F, 5, 2, 157-167

Chang, F. R., Li, M. S., Xu, P. P., Zhou, H. C., Haque, M. M., \& Huang, H. L. (2016). Injury Severity of Motorcycle Riders Involved in Traffic Crashes in Hunan, China: A Mixed Ordered Logit Approach. International Journal of Environmental Research and Public Health, 13, 7, 15.

de Craen, S., Doumen, M. J. A., \& van Norden, Y. (2014). A different perspective on conspicuity related motorcycle crashes. Accident Analysis and Prevention, 63, 133-137.

Clarke, D.D., Ward, P.J., Bartle, C., \& Truman, W. (2007). The role of motorcyclist and other driver behaviour in two types of serious accident in the UK. Accident Analysis and Prevention 39, 5 , 974-981.

Crundall, D., Bibby, P., Clarke, D.D., Ward, P., \& Bartle, C. (2008a). Car drivers' attitudes towards motorcyclists: a survey. Accident Analysis and Prevention 40, 3, 983-993.

Crundall, D., Clarke, D., Ward, P., \& Bartle, C. (2008b). Car Drivers'Skills and Attitudes to Motorcycle Safety. Department for Transport, London, ISBN 9781904763871. 
Crundall, D., Humphrey, K., \& Clarke, D. D. (2008c). Perception and appraisal of approaching motorcycles at junctions. Transportation Research Part F, 11, 159-167.

Crundall, D., Crundall, E., Clarke, D., \& Shahar, A. (2012). Why do car drivers fail to give way to motorcycles at T-junctions? Accident Analysis \& Prevention, 44, 1, 88-96.

Diamond, R., \& Carey, S. (1986). Why faces are and are not special: An effect of expertise. Journal of Experimental Psychology: General, 115, 2, 107-117.

Department for Transport (2016). Reported Road Casualties Great Britain: Main Results: 2015. Retrieved from https://www.gov.uk/government/uploads/system/uploads/attachment data/file/533293/rrc gb-main-results-2015.pdf, on 14/10/16.

European Commission (2015). Making roads safer for motorcycles and mopeds. Retrieved from http://ec.europa.eu/transport/road safety/users/motorcyclists-mopdeds, on 17/10/16.

Fiorentini, A., \& Berardi, N. (1981). Learning in grating waveform discrimination: Specificity for orientation and spatial frequency. Vision Research, 21, 7, 1149-1158.

Gauthier, I., \& Tarr, M. J. (1997). Becoming a "greeble" expert: Exploring mechanisms for face recognition. Vision Research, 37, 12, 1673-1682.

Gershon, P., Ben-Asher, N., \& Shinar, D. (2012). Attention and search conspicuity of motorcycles as a function of their visual context. Accident Analysis and Prevention, 44, 1, 97-103.

Gould, M., Poulter, D.R., Helman, S., Wann, J.P., (2012a). Errors in judging the approach rate of motorcycles in night-time conditions and the effect of an improved lighting configuration. Accident Analysis and Prevention, 45, 432-437.

Gould, M., Poulter, D.R., Helman, S., \& Wann, J.P. (2012b). Judgement of approach speed for motorcycles across different lighting levels and the effect of an improved tri-headlight configuration. Accident Analysis and Prevention, 48, 341-345.

Grill-Spector, K., \& Kanwisher, N. (2005). Visual recognition: As soon as you know it is there, you know what it is. Psychological Science, 16, 152-160.

Harel, A., Ullman, S., Harari, D., \& Bentin, S. (2011). Basic-level categorization of intermediate complexity fragments reveals top-down effects of expertise in visual perception. Journal of Vision, 11, 8, 18. 
Hershler, O., \& Hochstein, S. (2009). The importance of being expert: top-down attentional control in visual search with photographs. Attention, Perception and Psychophysics, 71, 7, 1478-1486.

Hills, B. L. (1980). Vision, visibility, and perception in driving. Perception, 9, 2, 183-216.

Horswill, M. S., \& Helman, S. (2003). A behavioral comparison between motorcyclists and a matched group of non-motorcycling car drivers: factors influencing accident risk. Accident Analysis and Prevention 35, 4, 589-597.

Husk, J. S., Bennett, P. J., \& Sekuler, A. B. (2007). Inverting houses and textures: Investigating the characteristics of learned inversion effects. Vision Research, 47, 27, 3350-3359.

IEEE (2012). Look Ma, no hands! Retrieved from http://www.ieee.org/about/news/2012/5september 2 2012.html, on 17/10/16.

Jackson, R. C., \& Farrow, D. (2005). Implicit perceptual training: How, when, and why? Human Movement Science, 24, 308-325.

Johnston, P., Brooks, C., \& Savage, H. (2008). Fatal and serious road crashes involving motorcyclists. Road Safety Research and Analysis Report. ATSB Monograph 20. Canberra: Department of Infrastructure, Transport, Regional Development and Local Government, 1-26.

Karni, A., \& Sagi, D. (1995). A memory system in the adult visual cortex. In B. Julesz \& I. Kovács (Eds.), Maturational windows and adult cortical plasticity. SFI Studies in the Sciences of Complexity. Vol. XXIV.

Kellman, P. J. (2002). Perceptual learning. In H.Pashler \& C. R.Gallistel (Eds.), Stevens' handbook of experimental psychology, 3rd ed., Vol. 3 (pp. 259-299). New York: John Wiley \& Sons.

Lee, Y. M., Sheppard, E., and Crundall, D. (2015). Cross-cultural effects on the perception and appraisal of approaching motorcycles at junctions. Transportation Research Part F, 31, 77-86

Levi, D. M., Knill, D. C., \& Bavelier, D. (2015). Stereopsis and amblyopia: A mini-review. Vision Research, 114, 17-30.

Macmillan, N. A., \& Kaplan, H. L. (1985). Detection theory analysis of group data: Estimating sensitivity from average hit and false-alarm rates. Psychological Bulletin, 98, 185-199. 
Magazzù, D., Comelli, M. \& Marinoni, A. (2006) Are car drivers holding a motorcycle licence less responsible for motorcycle-car crash occurrence? A nonparametric approach. Accident Analysis and Prevention, 38, 365-370.

Magill, R. A. (1998). Knowledge is more than we can talk about: Implicit learning in motor skill acquisition. Research Quarterly for Exercise and Sport, 69, 104-110.

McLaren, I.P.L. (1997). Categorization and perceptual learning: An analogue of the face inversion effect. The Quarterly Journal of Experimental Psychology 50A, 257-273

Myles-Worsley, M., Johnston, W.A., \& Simon, M.A. (1988). The influence of expertise on X-ray image processing. Journal of Experimental Psychology: Learning, Memory, and Cognition. 14, 553557.

National Highway Traffic Safety Administration (2007). Traffic safety facts 2005: Motorcycles. Washington, DC: NHTSA.

Oliva, A. \& Torralba, A. (2006). Building the Gist of a Scene: The Role of Global Image Features in Recognition. Progress in Brain Research, 155, 23-36

Pai, C. W., \& Saleh, W. (2008). Exploring motorcyclist injury severity in approach-turn collisions at Tjunctions: Focusing on the effects of driver's failure to yield and junction control measures. Accident Analysis \& Prevention, 40, 2, 479-486.

Pinto, M., Cavallo, V., \& Saint-Pierre, G. (2014). Influence of front light configuration on the visual conspicuity of motorcycles. Accident Analysis and Prevention, 62, 230-237.

Poggio, T., Fahle, M., \& Edelman, S. (1992). Fast perceptual learning in visual hyperacuity. Science, 256, 5059, 1018-1021.

Qin, X. A., Koutstaal, W., \& Engel, S. A. (2014). The hardwon benefits of familiarity in visual search: Naturally familiar brand logos are found faster. Attention, Perception, and Psychophysics, 76, 914-930.

Ranchet, M., Cavallo, V., Dang, N. T., \& Vienne, F. (2016). Improving motorcycle conspicuity through innovative headlight configurations. Accident Analysis and Prevention, 94, 119-126.

Reeder, R. R., Stein, T., \& Peelen, M. V. (2016). Perceptual expertise improves category detection in natural scenes. Psychonomic Bulletin and Review, 23, 172-179. 
Roy, M. M., \& Liersch, M. J. (2014). I am a better driver than you think: examining self-enhancement for driving ability. Journal of Applied Social Psychology, 43, 8, 1648-1659

Roge, J., El Zufari, V., Vienne, F., \& Ndiaye, D. (2015). Safety messages and visibility of vulnerable road users for drivers. Safety Science, 79, 29-38.

Rosenbloom T., Perlman A., \& Pereg A. (2011). Hazard perception of motorcyclists and car drivers. Accident Analysis and Prevention, 43, 601-604.

Sagi, D. (2011). Perceptual learning in Vision Research. Vision Research, 51, 1552-1566.

Scott, L. S., Tanaka, J. W., Sheinberg, D. L., \& Curran, T. (2006). A reevaluation of the electrophysiological correlates of expert object processing. Journal of cognitive Neuroscience, $18,9,1453-1465$.

Seaborn, K. \& Fels, D. I. (2015). Gamification in theory and action: A survey. International Journal of Human-Computer Studies, 74, 14-31.

Shaheed, M. S., Marshall, D., \& Gkritza, K. (2015). Factors Affecting Drivers' Ability to Detect Motorcycles During Daylight in a Simulated Environment. Transportation Research Record, $2520,143-150$.

Sowden, P. T., Davies, I. R., \& Roling. P. (2000). Perceptual learning of the detection of features in Xray images: a functional role for improvements in adults' visual sensitivity? Journal of Experimental Psychology: Human Perception and Performance, 26, 1, 379-390.

Stanislaw, H., \& Todorov, N. (1999). Calculation of signal detection theory measures. Behavior research methods, instruments, \& computers, 31, 1, 137-149.

Summala, H., Nieminen, T., \& Punto, M. (1996) Maintaining lane position with peripheral vision during in-vehicle tasks. Human Factors, 38, 3, 442-451.

Tanaka, J. W., \& Taylor, M. (1991). Object categories and expertise: Is the basic level in the eye of the beholder? Cognitive Psychology, 23, 457-482.

Walton, D., Buchanan, J., \& Murray, S. J. (2013). Exploring factors distinguishing car-versus-car from car-versus-motorcycle in intersection crashes. Transportation Research Part F, 17, 145-153.

Wilson, S., Darling, S., \& Sykes, J. (2011). Adaptive memory: fitness relevant stimuli show a memory advantage in a game of Pelmanism. Psychonomic Bulletin \& Review, 18, 4, 781-786. 
